\title{
Characterization of functional domains of the cblD (MMADHC) gene product
}

\author{
Jusufi, Jehona ; Suormala, Terttu ; Burda, Patricie ; Fowler, Brian ; Froese, D Sean ; Baumgartner,
} Matthias R

\begin{abstract}
In humans vitamin B12 (cobalamin, $\mathrm{Cbl}$ ) must be converted into two coenzyme forms, methylcobalamin $(\mathrm{MeCbl})$ and adenosylcobalamin (AdoCbl), in order to maintain intracellular homeostasis of homocysteine and methylmalonic acid, respectively. Previously we have shown that in cblD patients three types of MMADHC mutations exist: 1) null mutations N-terminal to Met116 cause isolated methylmalonic aciduria (cblD-MMA) due to AdoCbl deficiency; 2) null mutations across the C-terminus (p.Y140-R250) cause combined methylmalonic aciduria and homocystinuria (cblD-MMA/HC) due to AdoCbl and MeCbl deficiency; 3) missense mutations in a conserved C-terminal region (p.D246-L259) cause isolated homocystinuria (cblD-HC) due to MeCbl deficiency. To better understand the domain boundaries related to $\mathrm{MeCbl}$ formation, we made selected point mutations and C-terminal truncations in MMADHC and tested rescue of $\mathrm{MeCbl}$ and AdoCbl synthesis in immortalized cblD-MMA/HC patient fibroblasts. Testing 20 mutations (15 missense and five C-terminal truncations) across p.P154-S287 revealed the presence of a region (p.R197-D226) responsible for MeCbl synthesis, which gave a similar cellular phenotype as cblD-HC. Further, mutation of the polypeptide stretch between the new and patient defined regions (p.D226-D246) and directly C-terminal to the patient region (p.L259-R266), gave cellular phenotypes intermediate to those of cblD-HC and cblD-MMA/HC. Finally, C-terminal truncation of more than 20 amino acids resulted in a cblD-MMA/HC like cellular phenotype, while truncation of between ten and 20 amino acids resulted in a cblD-HC like cellular phenotype. These data suggest that specific regions of MMADHC are involved in differential regulation of AdoCbl and $\mathrm{MeCbl}$ synthesis and help better define the boundaries of these regions.
\end{abstract}

DOI: https://doi.org/10.1007/s10545-014-9709-4

Posted at the Zurich Open Repository and Archive, University of Zurich

ZORA URL: https://doi.org/10.5167/uzh-103315

Journal Article

Published Version

Originally published at:

Jusufi, Jehona; Suormala, Terttu; Burda, Patricie; Fowler, Brian; Froese, D Sean; Baumgartner, Matthias R (2014). Characterization of functional domains of the cblD (MMADHC) gene product. Journal of Inherited Metabolic Disease, 37(5):841-849.

DOI: https://doi.org/10.1007/s10545-014-9709-4 


\title{
Characterization of functional domains of the cblD (MMADHC) gene product
}

\author{
Jehona Jusufi • Terttu Suormala • Patricie Burda • \\ Brian Fowler • D. Sean Froese • Matthias R. Baumgartner
}

Received: 29 January 2014 / Revised: 14 March 2014 / Accepted: 17 March 2014 / Published online: 11 April 2014

(C) SSIEM and Springer Science+Business Media Dordrecht 2014

\begin{abstract}
In humans vitamin $\mathrm{B}_{12}$ (cobalamin, $\mathrm{Cbl}$ ) must be converted into two coenzyme forms, methylcobalamin (MeCbl) and adenosylcobalamin (AdoCbl), in order to maintain intracellular homeostasis of homocysteine and methylmalonic acid, respectively. Previously we have shown that in cblD patients three types of MMADHC mutations exist: 1) null mutations N-terminal to Met116 cause isolated methylmalonic aciduria ( $c b l D-M M A)$ due to AdoCbl deficiency; 2) null mutations across the C-terminus (p.Y140R250) cause combined methylmalonic aciduria and homocystinuria ( $c b l D-\mathrm{MMA} / \mathrm{HC}$ ) due to AdoCbl and $\mathrm{MeCbl}$ deficiency; 3) missense mutations in a conserved C-terminal region (p.D246-L259) cause isolated homocystinuria ( $c b l D-$ $\mathrm{HC})$ due to $\mathrm{MeCbl}$ deficiency. To better understand the domain boundaries related to $\mathrm{MeCbl}$ formation, we made selected point mutations and $\mathrm{C}$-terminal truncations in MMADHC and tested rescue of $\mathrm{MeCbl}$ and $\mathrm{AdoCbl}$ synthesis in
\end{abstract}

Communicated by: Verena Peters

Electronic supplementary material The online version of this article (doi:10.1007/s10545-014-9709-4) contains supplementary material, which is available to authorized users.

J. Jusufi $\cdot$ T. Suormala $\cdot$ P. Burda $\cdot$ B. Fowler $\cdot$ D. S. Froese $(\triangle) \cdot$

M. R. Baumgartner $(\bowtie)$

Division for Metabolic Disorders and Children's Research Center, University Children's Hospital, Steinweisstrasse 75, 8032 Zurich,

Switzerland

e-mail: Sean.Froese@kispi.uzh.ch

e-mail: matthias.baumgartner@kispi.uzh.ch

M. R. Baumgartner

radiz - Rare Disease Initiative Zurich, Clinical Research Priority

Program for Rare Diseases, University of Zurich, Zurich, Switzerland

M. R. Baumgartner

Zurich Center for Integrative Human Physiology,

University of Zurich, Zurich, Switzerland immortalized $c b l D-\mathrm{MMA} / \mathrm{HC}$ patient fibroblasts. Testing 20 mutations (15 missense and five C-terminal truncations) across p.P154-S287 revealed the presence of a region (p.R197-D226) responsible for MeCbl synthesis, which gave a similar cellular phenotype as $c b l D$-HC. Further, mutation of the polypeptide stretch between the new and patient defined regions (p.D226-D246) and directly C-terminal to the patient region (p.L259-R266), gave cellular phenotypes intermediate to those of $c b l D-\mathrm{HC}$ and $c b l D-\mathrm{MMA} / \mathrm{HC}$. Finally, C-terminal truncation of more than 20 amino acids resulted in a cblD$\mathrm{MMA} / \mathrm{HC}$ like cellular phenotype, while truncation of between ten and 20 amino acids resulted in a cblD-HC like cellular phenotype. These data suggest that specific regions of MMADHC are involved in differential regulation of AdoCbl and $\mathrm{MeCbl}$ synthesis and help better define the boundaries of these regions.

\section{Introduction}

Vitamin $\mathrm{B}_{12}$ (cobalamin; $\mathrm{Cbl}$ ) is needed in humans for two enzymes, mitochondrial methylmalonyl-CoA mutase (MUT: EC 5.4.99.2) which requires adenosylcobalamin and cytosolic methionine synthase (MS: EC 2.1.1.13) which requires methylcobalamin $(\mathrm{MeCbl})$. Deficiencies in either enzyme or the proteins required for the intracellular uptake and modification of cobalamin to these cofactor forms, designated as complementation groups cblA-G, cblJ and mut, result in homocystinuria (HC) for MS, methlymalonic aciduria (MMA) for MUT, or both (Baumgartner 2013; Froese and Gravel 2010). Subjects with mutations in the genes $M M A A$ (cblA) and $M M A B(c b l B)$ have decreased production of AdoCbl, resulting in MUT deficiency and isolated methylmalonic aciduria (Dobson et al 2002a; Dobson et al 2002b). Mutations in MTR ( $c b l G)$, the gene encoding MS (Chen et al 1997; Leclerc et al 1996; Li et al 1996), and 
MTRR ( $c b l E$ ), the gene encoding MS reductase (Leclerc et al 1998), result in decreased MeCbl production causing MS deficiency. Mutations in the genes ABCD4 (cblJ) (Coelho et al 2012), LMBRD1 (cblF) (Rutsch et al 2009) and $M M A C H C(c b l C)$ (Lerner-Ellis et al 2006) produce combined deficiency of MUT and MS, resulting in both methylmalonic aciduria and homocystinuria. Finally, the $c b l D$ complementation group is unique in that it can be associated with isolated methylmalonic aciduria ( $c b l D$ MMA), isolated homocystinuria ( $c b l D-H C)$, or combined methylmalonic aciduria and homocystinuria (cblD-MMA/ HC) (Suormala et al 2004). Clinical presentation of the three cblD patient groups largely follows that of the other complementation groups (Baumgartner 2013), with $c b l D$-MMA/ $\mathrm{HC}$ and $c b l D-\mathrm{HC}$ patients displaying neurological and haematological symptoms, while $c b l D$-MMA patients have respiratory distress and hyperammonemia as well as neurological symptoms (Coelho et al 2008; Parini et al 2013; Suormala et al 2004).

The type and location of patient mutations across the MMADHC protein, responsible for $c b l D$, correspond to disease phenotype (Coelho et al 2008). Truncation mutations towards the N-terminus of the protein on at least one of the two mutant alleles result in $c b l D-M M A$, truncation or missense mutations towards the C-terminus result in cblD$\mathrm{MMA} / \mathrm{HC}$, and missense mutations in a narrow region near the C-terminus (p.D246-L259) result in cblD-HC (Stucki et al 2012). The MMA only phenotype of N-terminal null mutations has been explained by reinitiation of translation at Met62 and Met116 (Stucki et al 2012). Thus, truncation mutations before Met116 ablate full-length protein production but allow the formation of a shortened protein, containing 180 amino acids, which appears to be sufficient for $\mathrm{MeCbl}$ synthesis. Additional studies (Stucki et al 2012) have shown that replacement of the endogenous mitochondrial leader sequence (MLS) with a more efficient one (from ALDH2) increases the proportion of cellular AdoCbl synthesis compared to $\mathrm{MeCbl}$, suggesting that the relatively weak endogenous MLS of MMADHC helps balance the production of the two cofactors. Interestingly, addition of this strong mitochondrial leader to a construct starting at Met116 resulted in almost no AdoCbl production, suggesting that amino acids N-terminal to Met116 are required for AdoCbl synthesis.

Despite this detailed information on the N-terminus of MMADHC and its relation to AdoCbl production, very few insights into the importance of the $\mathrm{C}$-terminus or requirements for $\mathrm{MeCbl}$ generation have been found. Our work aims to better understand these functions. Using site directed mutagenesis (SDM) to introduce 15 missense mutations between p.154-280, along with five C-terminal mutations between p.261-287, we examined the effects these mutations had on AdoCbl and especially MeCbl synthesis in an immortalized $c b l D$-HC/MMA cell line. Our results suggest distinct $\mathrm{C}$-terminal protein regions control MMADHC targeted $\mathrm{MeCbl}$ synthesis, while other regions play important roles in the synthesis of both cofactors.

\section{Materials and methods}

\section{Cloning}

Untagged full-length wild-type $M M A D H C$ cloned into the pTracer-CMV2 expression vector (pTracer-MMADHC-wt) has been previously described (Stucki et al 2012). All missense and truncation mutants were prepared on this pTracerMMADHC-wt construct using the Quikchange site-directed mutagenesis kit (Stratagene), following manufacturer's protocols. Sequences of forward and reverse primers (Microsynth) used to generate the mutations are given in Supplementary Table 1. All mutant constructs were confirmed by Sanger sequencing. Plasmid DNA for transfections were prepared using the QIAfilter Plasmid Maxi Kit (Qiagen) and DNA concentration determined by spectrophotemtry (Nanodrop 2000; Thermo Scientific).

\section{Cell culture and expression}

Transformed fibroblasts of the $c b l D-M M A / H C$ patient D007 (Coelho et al 2008), grown in routine Dulbecco's Modified Eagle's Medium (DMEM; Gibco) supplemented with $10 \%$ foetal calf serum (FCS; Gibco) and antibiotics (PAA), were used for transfections throughout the study. Expression of pTracer, MMADHC-wt and mutant constructs were performed as earlier described for the study of MMADHC constructs (Coelho et al 2008; Stucki et al 2012).

\section{Cobalamin coenzyme synthesis assay}

Synthesis of cobalamin coenzymes was estimated using a method modified (Fowler and Jakobs 1998; Suormala et al 2004) from the original (Mahoney and Rosenberg 1971). For a more complete description of the procedure see Supplementary methods.

\section{Statistics}

All values come from at least three independent experiments, with statistical significance determined using an unpaired twotailed Student's T-test with a cut-off of $P<0.05$, unless otherwise indicated. Statistics were performed using GraphPad Prism v6 software. 


\section{Results}

Rationale for mutation selection

Three patient mutations on MMADHC - p.D246G, p.Y249C and p.L259P - are known to cause the cblD$\mathrm{HC}$ phenotype and thus interfere with proper $\mathrm{MeCbl}$ formation. To better understand the boundaries of the region responsible for $\mathrm{MeCbl}$ production, we used SDM to substitute residues across the middle and $\mathrm{C}$-terminus of the MMADHC protein and tested them for synthesis of $\mathrm{MeCbl}$ and AdoCbl. In all, we mutated 15 residues, each to alanine, ranging from p.P154 to p.D286, choosing to substitute those amino acids after Met116 that were strongly conserved and represented distinct regions of conservation across the polypeptide (Fig. 1). Of the 15 residues mutated, all but four (p.E167, p.F204, p.R266, p.S278) were completely conserved down to $C$. elegans (Fig. 1). In addition, we generated five truncation mutations between p.C261 and p.S287 (Fig. 1) in order to test the importance of the C-terminus for the function of the protein.
Generation of $\mathrm{MeCbl}$ and $\mathrm{AdoCbl}$ is dependent on the construct expressed

Figure 2 shows representative graphs of the separation of labelled-cobalamins by HPLC after transfection with vector only (A), MMADHC-wild-type (B) and MMADHC-L259P (C), a cblD-HC patient mutation. When immortalized cblD-MMA/HC patient fibroblasts were transfected with pTracer vector only, the majority of cobalamins detected were in the $\mathrm{OHCbl}$ form, with very minor peaks for CNCbl, AdoCbl and MeCbl (Fig. 2a). These cells were therefore able to convert $\mathrm{CNCbl}$ to $\mathrm{OHCbl}$, most likely through the action of MMACHC (Kim et al 2008), but unable to process $\mathrm{Cbl}$ further, biochemically consistent with the $c b l D-M M A / H C$ phenotype. After expression of the MMADHC-wild-type vector, there was a marked increase in $\mathrm{MeCbl}$ formation, but a less prominent AdoCbl peak (Fig. 2b), suggesting that the rescue of $\mathrm{MeCbl}$ and AdoCbl is uneven, consistent with previously published results (Coelho et al 2008; Stucki et al 2012). Following over-expression of MMADHC-L259P, there was only a slight increase in $\mathrm{MeCbl}$, but a large
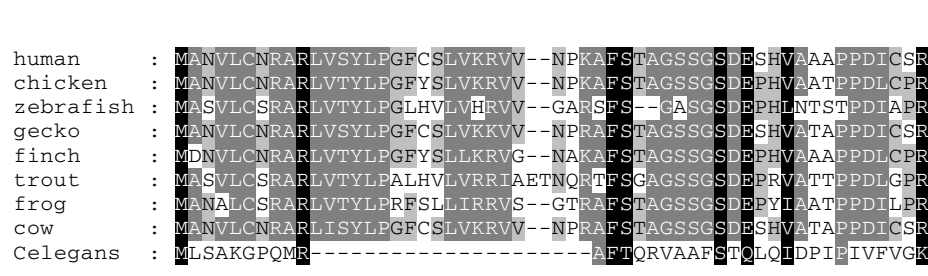

Met62
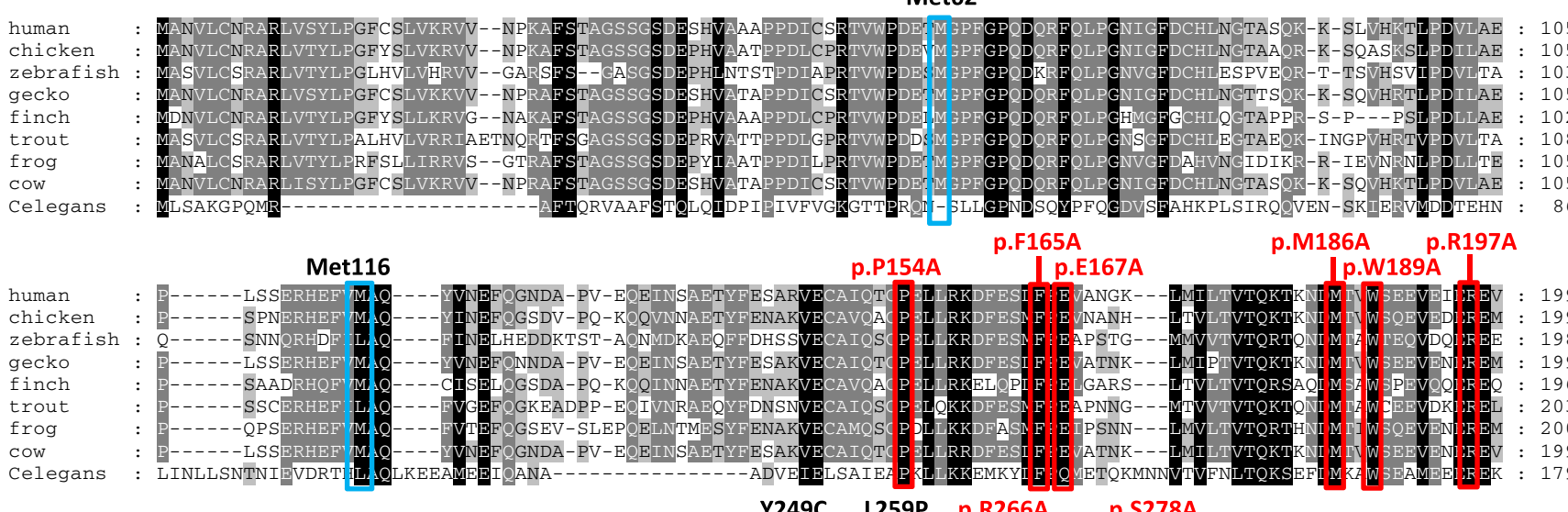

p.F204A p.C212A

p.D226A p.Y237A D246G

Y249C L259P p.R266A p.S278A

$\begin{array}{ll}\text { human } & : \\ \text { chicken } & : \\ \text { zebrafish } & : \\ \text { gecko } & : \\ \text { finch } & : \\ \text { trout } & : \\ \text { frog } & : \\ \text { cow } & :\end{array}$
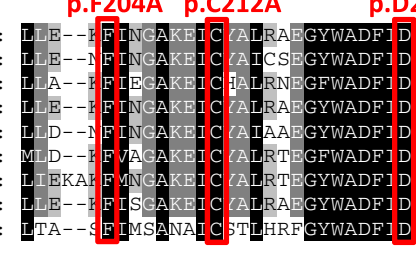

Fig. 1 Multiple sequence alignment of MMADHC across evolution with mutations identified. Residues that are identical in all species listed are shown in black; residues with conservative substitutions are shown in grey. Met62 and Met116 (blue), cblD-HC patient mutations (yellow), amino acids used for missense mutations (red boxes) and those for $\mathrm{C}$ terminal truncations (green lines) are shown. Sequences are shown for human (Homo sapiens: NP_056517), chicken (Gallus gallus:

NP 001008477), zebrafish (Danio rerio: NP 991157), gecko (Gekko japonicus: Q6DV04), finch (Geospiza fortis: XP_005415776), trout (Oncorhynchus mykiss: ACO07932), frog (Xenopus laevis: NP_001089489), cow (Bos Taurus: NP_001029713) and C. elegans (Caenorhabditis elegans NP_499801). This alignment was generated by Tcoffee (tcoffee.org) and visualized using GeneDoc (Nicholas et al 1997) 

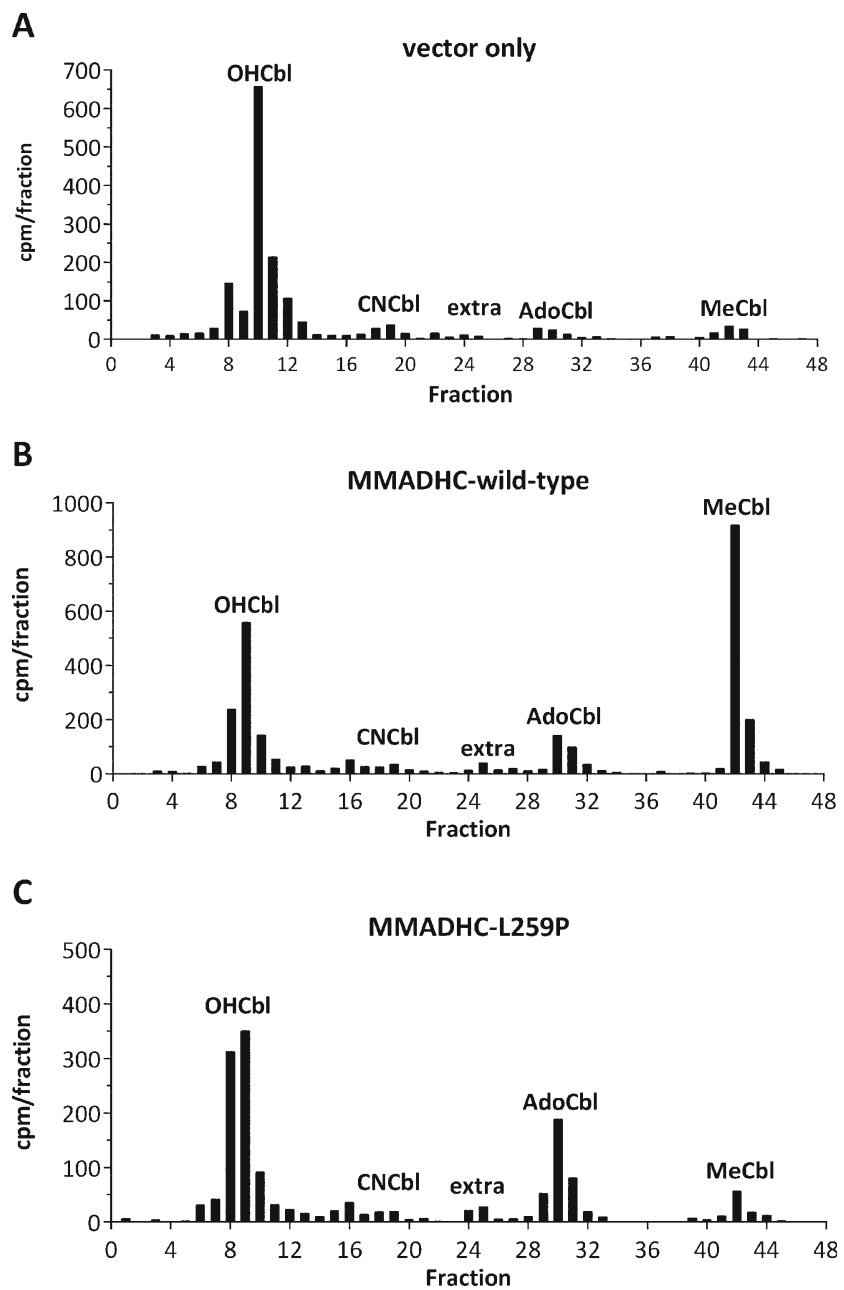

Fig. 2 HPLC separation of cobalamin derivatives in extracts. The cobalamin coenzyme synthesis assay was used to estimate the ability of transfected cells to synthesise adenosylcobalamin (AdoCbl) and methylcobalamin $(\mathrm{MeCbl})$. This separation technique was modified from the original method (see Supplementary methods) to separate AdoCbl from an unknown radioactive compound eluting at 23-26 min (extra). Panels a-c show a representative distribution of radioactivity in cells transfected with an empty $p$ Tracer vector $(\mathbf{a})$, p Tracer constructs containing MMADHC-wild-type (b), and MMADHC-L259P, a cblD-HC patient mutation (c). The retention times of hydroxocobalamin $(\mathrm{OHCbl}), \mathrm{CNCbl}$, $\mathrm{AdoCbl}$ and $\mathrm{MeCbl}$ were estimated by detecting unlabelled compounds added to the cell pellet before extraction (each $20 \mu \mathrm{g} /$ pellet) at $254 \mathrm{~nm}$. cpm: counts per minute

increase in AdoCbl, compared to vector only control (Fig. 2c). This is consistent with a cellular cblD-HC phenotype, and together with the MMADHC-wild-type result, is compatible with the hypothesis that MMADHC targeted $\mathrm{Cbl}$ processing to either $\mathrm{MeCbl}$ or AdoCbl is delicately balanced, and an increase in the production of one cofactor form results in a concomitant decrease in the other. There was no significant difference in the total radioactive counts for any of these controls, suggesting that cellular cobalamin uptake was not affected by the presence or absence of MMADHC protein.
Effects on AdoCbl and Mecbl synthesis by MMADHC SDM missense mutations

Testing our constructs with the generated missense mutations gave a spectrum of results, including no correction of either AdoCbl or $\mathrm{MeCbl}$, correction of both to wild-type levels, or correction of AdoCbl but not $\mathrm{MeCbl}$ as in cblD-HC (Fig. 3a; Table 1). Comparison of the results of $\mathrm{AdoCbl}$ and $\mathrm{MeCbl}$ production for each mutation with each of our controls (Table 1) allowed grouping of the mutations into categories, highlighting their similarity and the nature of the expected cellular phenotype if these mutations manifested in patients (Fig. 3a). Where a particular mutation led to no significant difference (Table 1, ns) in levels of AdoCbl and $\mathrm{MeCbl}$ compared with vector-only, wild-type or cblD$\mathrm{HC}$, that mutation was placed in the same category as that control. Where one or both of AdoCbl and $\mathrm{MeCbl}$ differed from all three controls, separate categories were created (see Supplementary results). Only one mutation,
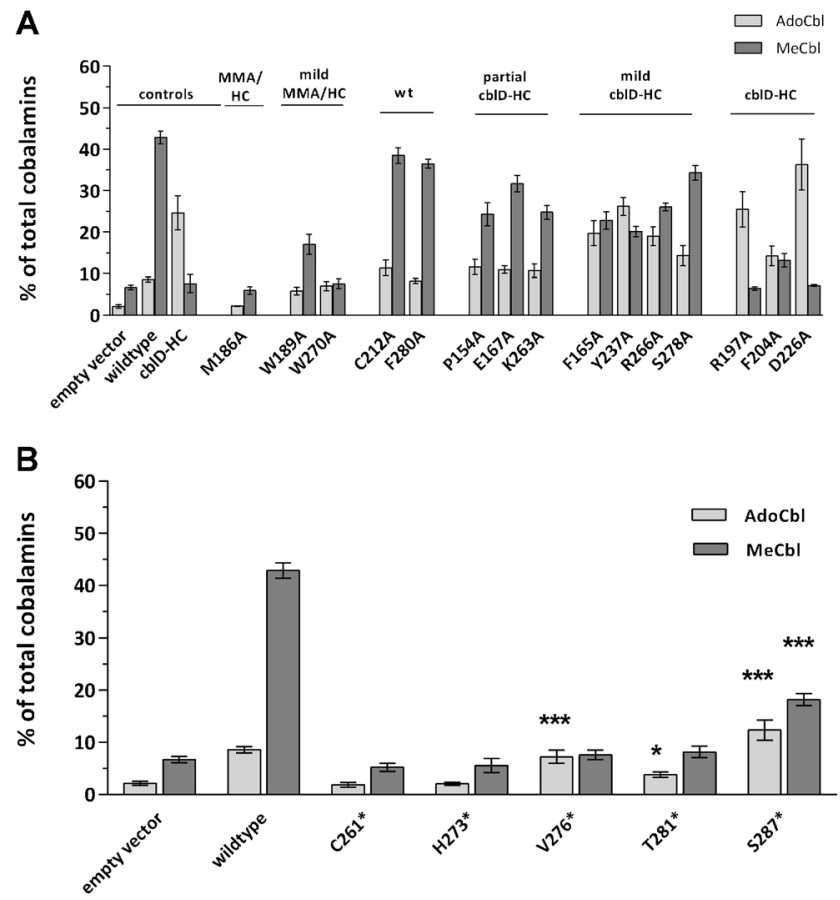

Fig. 3 AdoCbl (light grey) and MeCbl (dark grey) synthesis in (a) missense and (b) C-terminal truncating mutations. a Mutations are grouped according to their phenotype from left to right in "controls" "combined $c b l D-M M A / H C "$, "mild $c b l D-M M A / H C "$, "wild-type" (wt), "partial $c b l D-H C$ ", "mild $c b l D-H C$ " and "isolated $c b l D-H C$ ". b Statistics performed for each mutation against empty vector are shown.

${ }^{*} P<0.05 ; * * P<0.005 ; * * * P<0.0001$. For both $\mathbf{A}$ and $\mathbf{B}$. Transfections with empty vector, MMADHC-wild-type and MMADHC-L259P were used as controls in each experiment. Each mutant construct was transfected in at least three independent experiments. The distribution of both $\mathrm{Cbl}$ derivatives is expressed as \% total cobalamins. Columns represent mean values of $\mathrm{AdoCbl}$ and $\mathrm{MeCbl}$ synthesis, error bars represent mean $+/-$ SD 
Table 1 Statistical analysis of each transiently expressed mutation against corresponding controls. Statistics were performed using an unpaired t-test (two-tailed), where all mutations were separately tested against each control (empty vector, wild-type and the $c b l D$-HC mutation p.L259P) for adenosylcobalamin (AdoCbl) and methylcobalamin (MeCbl), respectively. Categorization of mutations was determined by these comparisons (see Supplementary results). For clarity, truncation mutations are represented as " $\mathrm{X}$ " instead of "*”. ns: not significant. $* P<0.05 ; * * P<0.005 ; * * * P<0.0001$, where dark background represents higher than the control, light background represents lower than the control. GraphPad Prism 6 software was used for analysis. All mutations were tested in at least three independent experiments

\begin{tabular}{|c|c|c|c|c|c|c|}
\hline & \multicolumn{3}{|c|}{ AdoCbl } & \multicolumn{3}{|c|}{ MeCbl } \\
\hline & empty vector & wildtype & cblD-HC (L259P) & empty vector & wildtype & cblD-HC (L259P) \\
\hline \multicolumn{7}{|c|}{ Missense Mutations } \\
\hline P154A & 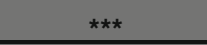 & ns & * & $\star \star \star *$ & $\star \star \star$ & $\star *$ \\
\hline F165A & 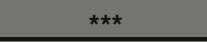 & *** & ns & $* * *$ & $\star * * *$ & ** \\
\hline E167A & *** & ns & * & *** & ** & $* * *$ \\
\hline M186A & ns & $\star \star \star$ & ** & ns & *** & ns \\
\hline W189A & $\star \star \star *$ & * & $\star \star \star$ & $\star \star \star *$ & $\star \star \star$ & * \\
\hline R197A & *** & $\star \star *$ & ns & ns & *** & ns \\
\hline F204A & $\star \star \star *$ & ** & ns & *** & $\star \star \star$ & ns \\
\hline C212A & $\star \star \star *$ & ns & * & *** & ns & *** \\
\hline D226A & $\star \star \star *$ & $* \star \star$ & ns & ns & 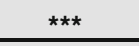 & ns \\
\hline Y237A & 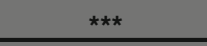 & $* * *$ & ns & $\star \star \star *$ & 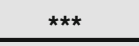 & ** \\
\hline K263A & 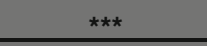 & ns & * & $\star \star \star *$ & 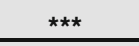 & *** \\
\hline R266A & $* * \star$ & *** & ns & *** & $\star \star \star *$ & *** \\
\hline W270A & $\star * \star *$ & ns & * & ns & 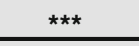 & ns \\
\hline S278A & $\star \star \star *$ & ** & ns & 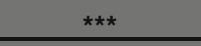 & ** & 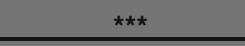 \\
\hline F280A & 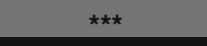 & ns & * & $\star \star \star$ & ns & $\star \star \star *$ \\
\hline \multicolumn{7}{|c|}{ C-terminal Truncations } \\
\hline C261X & ns & $\star \star \star *$ & ** & ns & $\star \star \star$ & ns \\
\hline $\mathrm{H} 273 \mathrm{X}$ & ns & $* \star \star$ & ** & ns & $* \star \star$ & ns \\
\hline V276X & $\star \star \star *$ & ns & ** & ns & $\star \star \star \star$ & ns \\
\hline T281X & * & $\star \star \star \star$ & ** & ns & 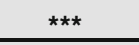 & ns \\
\hline S287X & $\star \star \star \star$ & * & * & $* * *$ & $\star * * *$ & ** \\
\hline
\end{tabular}

p.M186A, failed to increase either AdoCbl or MeCbl compared to empty vector control and was therefore placed in the combined "cblD-MMA/HC" category. Two mutations, p.C212A and p.F280A, gave results consistent with addition of wild-type vector, suggesting that even though these mutations were introduced at strictly conserved residues (Fig. 1), their substitution did not affect protein function. Resequencing of both constructs confirmed their correct mutation (data not shown). Three mutations, falling within 30 amino acids of each other - p.R197A, p.F204A and p.D226A - produced AdoCbl and $\mathrm{MeCbl}$ levels consistent with the " $c b l D-H C$ " cellular phenotype. The other nine mutations gave cellular phenotypes intermediate to these previously identified categories. p.W189A and p.W270A each showed slightly increased AdoCbl levels, while p.W189A also had slightly increased MeCbl levels, compared to empty vector, but they remained low compared to wild-type enzyme. These were therefore categorized as "mild $c b l D$ -
MMA/HC". The next group (p.P154A, p.E167A, p.K263A) showed increases in AdoCbl similar to wildtype, but reduced $\mathrm{MeCbl}$ production. To reflect this pattern, they were categorized as "partial cblD-HC". Finally, the group defined as "mild cblD-HC" (p.F165A, p.Y237A, p.R266A and p.S278A) showed similar MeCbl but elevated AdoCbl levels compared to the "partial cblD-HC" group. Indeed, their AdoCbl levels were not-significantly different from those generated by the cblD-HC mutations; however, their MeCbl levels were much higher, suggesting they might cause a very "mild $c b l D-H C$ " like phenotype, if any.

Effects on AdoCbl and MeCbl synthesis by MMADHC C-terminal truncations

To test the importance of the C-terminus to the overall function of the protein, we constructed five C-terminal 
truncation mutations, including p.C261*, p.H273*, p.V276*, p.T281* and S287*, shortening the 296 amino acid full-length protein by between nine and 36 amino acids (Fig. 1). We found that for every truncation except p.S287*, there was no significant increase in MeCbl production compared to vector only (Fig. 3b). Alternatively, while AdoCbl production was not rescued in p.C261* and p.H273*, it was slightly, but significantly, increased in p.V276* and p.T281* and increased almost four-fold for p.S287* compared to empty vector (Fig. 3b). These results suggest that $\mathrm{C}$-terminal truncations up to p.H273 result in completely non-functional protein, while truncations between p.V276 and p.S287 result in a protein that is partially able to rescue AdoCbl but not $\mathrm{MeCbl}$ synthesis. Truncations from p.S287, incorporating all but the last nine amino acids, result in a protein that can partially rescue both AdoCbl and $\mathrm{MeCbl}$ synthesis and therefore is minimally functional for both coenzyme synthesis pathways.

\section{Discussion}

Importance of $\mathrm{N}$ - and $\mathrm{C}$-terminal regions to $\mathrm{AdoCbl}$ and $\mathrm{MeCbl}$ production

Since the identification of the gene responsible for the cblD defect, $M M A D H C$, much knowledge about its protein's function has come from mutation analysis. The gene identification paper (Coelho et al 2008) described an initial genotype-phenotype relationship which has held up well with the identification of an additional 12 patients and six mutations (Parini et al 2013; Stucki et al 2012). Thus far, all truncating mutations described N-terminal to p.N77 have resulted in cblDMMA (Fig. 4a solid light blue) while all truncating mutations after p.Y140 resulted in $c b l D-M M A / H C$. Further work has shown that deletion of the N-terminal 115 amino acids leads to significantly decreased AdoCbl production (Fig. 4a all light blue) with almost no decrease in $\mathrm{MeCbl}$ formation, reinforcing the importance of the N-terminus for AdoCbl production and the cblDMMA phenotype, while demonstrating its low importance for MeCbl synthesis (Stucki et al 2012). We therefore focused our attention on the C-terminus, examining the effects of missense and truncating mutations across this part of the protein on the synthesis of AdoCbl and $\mathrm{MeCbl}$.

We found that truncations of greater than 23 amino acids resulted in no significant increase in formation of either coenzyme compared with empty vector, indicating lack of function of the generated protein. Truncations of less than 20 amino acids resulted in AdoCbl production, but truncation of only 15 amino acids still resulted in no $\mathrm{MeCbl}$ production. This suggests that the C-terminal residues are very important for conversion of cobalamin into the $\mathrm{MeCbl}$ form, but less so for AdoCbl. This data is complementary to the N-terminal truncations produced by Stucki et al (2012). It also demonstrates that the $\mathrm{C}$-terminus is far less tolerant to truncation than the $\mathrm{N}$-terminus. More than $60 \mathrm{~N}$-terminal amino acids can be cleaved without loss of function to either pathway, but deletion of just 23 residues from the C-terminus is detrimental to production of both cofactors. This is also reflected in the much stronger conservation of the protein's C-terminus across all species examined (Fig. 1). Taken together these data are consistent with an important role for the targeting of Cbl towards the mitochondria by the N-terminus of MMADHC and distribution towards the cytosol governed by the $\mathrm{C}$-terminus.

\section{Identification of a further $c b l D-\mathrm{HC}$ causing region}

Generation of missense mutations across the C-terminal half of the protein identified a spectrum of phenotypes related to the production of $\mathrm{AdoCbl}$ or $\mathrm{MeCbl}$. While we found large differences in $\mathrm{MeCbl}$ and AdoCbl levels for different mutations, one or both cofactor(s) were elevated above vector only levels (Fig. 3a, Table 1) in all but one case, suggesting that a partially functioning protein for each mutant was produced. Only one of the residues we mutated was found in the 1000 Genomes genetic variation database; p.M186, at a frequency of < 0.001 ; while no protein changing polymorphisms within the entire investigated region were found above an allele frequency of 0.006 , suggesting an importance of this region to protein function.

From these mutations we identified a second region of the protein, in addition to that delineated by patient mutations, where mutation resulted in a cellular phenotype consistent with cblD-HC (Fig. 4a - solid dark blue). This region included three mutations (p.R197A, p.F204A and p.D226A) which gave the $c b l D-H C$ phenotype, with one mutation (p.C212A) in between, anomalously, giving wild-type. Although p.C212 is strongly conserved (Fig. 1), and p.C212A was predicted by mutational effect prediction sites (SIFT: http://www. sift.jcvi.org; PolyPhen2: http://www.genetics.bwh. harvard.edu/pph2) to be "probably damaging" (SIFT: 0. 00-0.01, Polyphen2: 0.975-0.998), it seems that this residue is tolerant to substitution. Together, our SDM delineated region (p.R197-D226) and the patient mutation region (p. D246-p.L259) represent important protein sites which appear to be necessary for the production of $\mathrm{MeCbl}$ but not AdoCbl. Therefore, it is possible that they correspond to important protein-protein interaction domains specific to 
A

\section{Patient mutations}

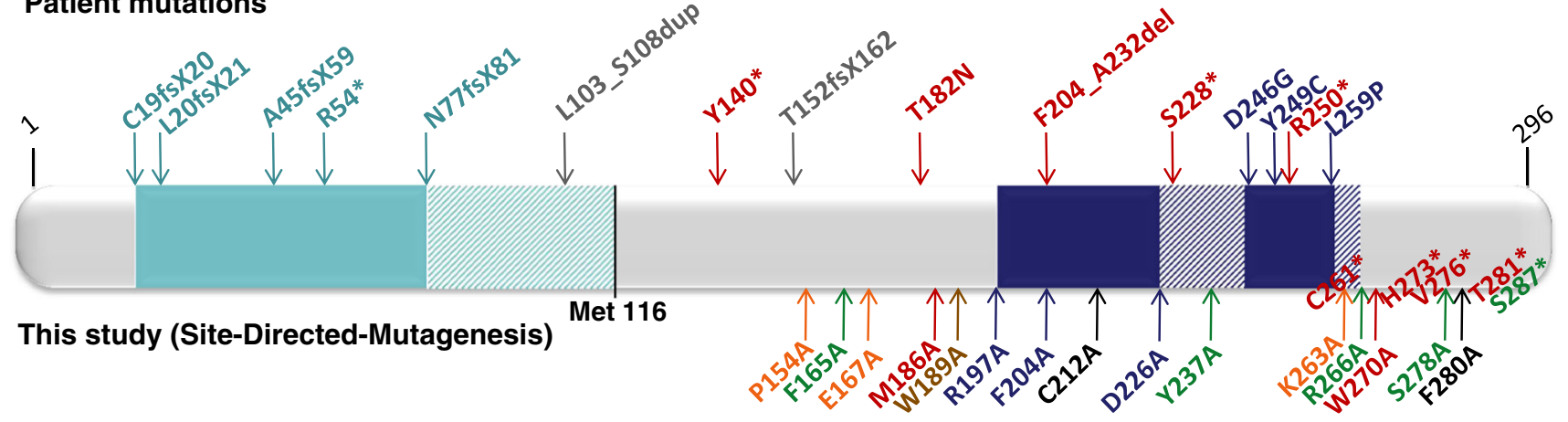

B

\section{Predicted secondary structure}

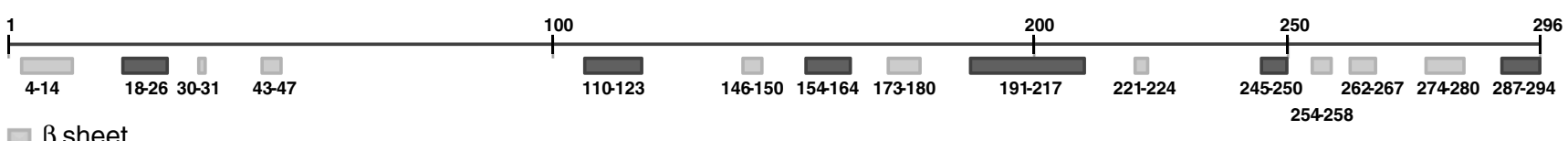

helix

Fig. 4 Amino acid sequence and predicted secondary structure of the MMADHC protein. a Schematic representation of MMADHC across all 296 amino acids. Mutations described in patients with $c b l D$ deficiency are shown above the protein; mutations generated by SDM are shown below. Light blue area: region where mutations cause $c b l D$-MMA; striped light blue: additional region responsible for AdoCbl synthesis; Met116:

cytosolic $\mathrm{MeCbl}$ targeting proteins. We also noted cellular phenotypes similar to that of $c b l D-\mathrm{HC}$ from mutations in the amino acid stretch between these $c b l D-\mathrm{HC}$ regions and just $\mathrm{C}$ terminal to the patient bound region (Fig. $4 \mathrm{a}$ - dark blue crosshatch). However, mutations in these areas produced higher $\mathrm{MeCbl}$ levels than seen in the $c b l D$-HC regions, suggesting a lesser ability to knock-out $\mathrm{MeCbl}$ formation, perhaps due to non-direct involvement with cytosolic targeting proteins.

Some of the missense mutations we generated resulted in severe impairment in intracellular cobalamin metabolism. For example, p.M186A showed no elevation of MeCbl or AdoCbl, while p.W189A gave only slightly increased MeCbl and AdoCbl above background levels (Fig. 3a). It is striking that these residues lie very close to the only known patient missense mutation that causes combined deficiency, p.T182N, suggesting this area is critical for both cobalamin cofactor pathways. Alternatively, the severity of these mutations could be due to misfolding.

Mapping secondary structure onto the mutation analysis reveals possible $\alpha$-helices and $\beta$-sheets important for functional interactions

It is interesting to speculate how these results might relate to the structure of MMADHC. In the absence of a protein crystal reinitiation of translation at Methionine 116; dark blue: region where mutations cause $c b l D-\mathrm{HC}$; striped dark blue: region where missense mutations cause a milder $c b l D-H C$ phenotype. B. The predicted secondary structure (http://www.predictprotein.org) of MMADHC with $\beta$ sheets (light grey boxes) and $\alpha$-helices (dark grey boxes) shown

structure, or even an appropriate homology model, we are presently limited to mapping these mutations onto its predicted secondary structure (Fig. 4b). Nevertheless some intriguing comparisons can be drawn. For example, the C-terminus of the protein is predicted to end in a $\alpha$-helix (aa 287-294), which is preceded by a $\beta$-sheet (aa 274-280). Deletion of the final $\alpha$-helix, which we achieved by the S287* construct, results in decreased $\mathrm{MeCbl}$ production, but normal AdoCbl, while truncation of both the $\alpha$-helix and $\beta$-sheet, by $\mathrm{H} 273^{*}$, results in no $\mathrm{MeCbl}$ or AdoCbl formation. This suggests that the final $\alpha$-helix is important for $\mathrm{MeCbl}$ but not AdoCbl formation. Another interesting area of secondary structure governs the $c b l D-\mathrm{HC}$ regions. The patient defined region is spanned by an $\alpha$-helix (aa 245-250) and a $\beta$-sheet (aa 254258). Our mutations (p.K263A and p.R266A) in the $\beta$-sheet immediately $\mathrm{C}$-terminal to this region (aa 262-267) resulted in a non-exclusive $c b l D$-HC phenotype. Likewise, an $\alpha$-helix (aa 191-217) and a $\beta$-sheet (aa 221-224) span the region of our SDM mutations which resulted in the $c b l D-H C$ phenotype, but mutations in the disordered or loop regions both $\mathrm{N}$ (p.W189A) or C- (p.Y237A) terminal to these regions of secondary structure gave mixed phenotypes. These data suggest that the two $\alpha$-helices and $\beta$-sheets spanning amino acids 191-258 constitute an area specific for the MeCbl synthesis pathway, with the loop/disordered regions between and on 
either side of them not being directly involved. It is possible that in 3-dimensional space, these secondary structure elements make up one contiguous area. This supports the idea that this area is directly involved with protein-protein interactions in the cytosolic only pathway.

Interaction with MMACHC and implications

Since MMADHC has not been found to bind cobalamin (Deme et al 2012), the most likely explanation of its cobalamin distribution function is via interaction with MMACHC, a protein shown to bind and modify cobalamin (Hannibal et al 2009; Kim et al 2008). Indeed, three papers have now shown interaction between these two proteins (Deme et al 2012; Gherasim et al 2013; Plesa et al 2011). By using phage display, Plesa et al (Plesa et al 2011) described five possible MMACHC interacting regions (I-V) of MMADHC. Of these, region III (p.Y220-T238) partially overlaps with our SDM region found to cause $c b l D$-HC, region IV (p.T245-G260) completely overlaps with the patient defined $c b l D$-HC region, and the 22 amino acids which make up region V (p.V274-L293) correspond to a region we found to be required for $\mathrm{MeCbl}$ but not AdoCbl formation from our truncation experiments. Thus the MMADHC regions we have identified as being key to $\mathrm{MeCbl}$ synthesis may correspond to those areas responsible for interaction with MMACHC. In this scenario, the interaction between MMACHC and MMADHC will target cobalamin to the cytosolic pathway, with eventual participation of methionine synthase. In support of this theory, MMACHC has recently been shown not to localize to the mitochondria (Mah et al 2013) and the N-terminal 116 amino acids of MMADHC are not required for interaction with MMACHC (Gherasim et al 2013) suggesting a lack of involvement of MMACHC in the AdoCbl processing pathway. Regardless of the crucial role of MMACHC in the early part of the $\mathrm{Cbl}$ synthesis pathway, our data is consistent with lack of involvement of MMACHC in the MMADHC directed part of the AdoCbl pathway.

\section{Conclusion}

In conclusion, we have found a unique protein region responsible for $\mathrm{MeCbl}$ but not $\mathrm{AdoCbl}$ synthesis, and defined the limits of Cterminal truncations that affect protein function. Our results suggest distinct protein regions are responsible for $\mathrm{MeCbl}$ production, and point to potential $\alpha$-helices and $\beta$-sheets as governing these regions. Further studies, addressing protein-protein interactions between MMADHC and MMACHC as well as others, using the regions determined here may give further insight into the complete process of $\mathrm{MeCbl}$ cofactor synthesis while the elucidation of the protein structure of MMADHC would be invaluable to understand how these regions and potential interactions are governed.
Acknowledgments This work was supported by the Swiss National Science Foundation [grant number 31003A_138521 to M.R.B. and B.F.] and the Rare Disease Initiative Zurich (radiz), a clinical research priority program for rare diseases of the University of Zurich, Switzerland.

Conflict of interest None.

\section{References}

Baumgartner MR (2013) Vitamin-responsive disorders: cobalamin, folate, biotin, vitamins B1 and E. Handb Clin Neurol 113:1799-1810

Chen LH, Liu ML, Hwang HY, Chen LS, Korenberg J, Shane B (1997) Human methionine synthase. cDNA cloning, gene localization, and expression. J Biol Chem 272:3628-3634

Coelho D, Suormala T, Stucki M et al (2008) Gene identification for the cblD defect of vitamin B12 metabolism. N Engl J Med 358:1454 1464

Coelho D, Kim JC, Miousse IR et al (2012) Mutations in ABCD4 cause a new inborn error of vitamin B12 metabolism. Nat Genet 44:11521155

Deme JC, Miousse IR, Plesa M et al (2012) Structural features of recombinant MMADHC isoforms and their interactions with MMACHC, proteins of mammalian vitamin B12 metabolism. Mol Genet Metab 107:352-362

Dobson CM, Wai T, Leclerc D et al (2002a) Identification of the gene responsible for the cblB complementation group of vitamin B12dependent methylmalonic aciduria. Hum Mol Genet 11:3361-3369

Dobson CM, Wai T, Leclerc D et al (2002b) Identification of the gene responsible for the cblA complementation group of vitamin B12responsive methylmalonic acidemia based on analysis of prokaryotic gene arrangements. Proc Natl Acad Sci U S A 99:15554-15559

Fowler B, Jakobs C (1998) Post- and prenatal diagnostic methods for the homocystinurias. Eur J Pediatr 157(Suppl 2):S88-S93

Froese DS, Gravel RA (2010) Genetic disorders of vitamin B metabolism: eight complementation groups-eight genes. Expert Rev Mol Med 12:e37

Gherasim C, Hannibal L, Rajagopalan D, Jacobsen DW, Banerjee R (2013) The C-terminal domain of CblD interacts with $\mathrm{CblC}$ and influences intracellular cobalamin partitioning. Biochimie 95:10231032

Hannibal L, Kim J, Brasch NE, Wang S, Rosenblatt DS, Banerjee R, Jacobsen DW (2009) Processing of alkylcobalamins in mammalian cells: a role for the MMACHC (cblC) gene product. Mol Genet Metab 97:260-266

Kim J, Gherasim C, Banerjee R (2008) Decyanation of vitamin B12 by a trafficking chaperone. Proc Natl Acad Sci U S A 105:14551-14554

Leclerc D, Campeau E, Goyette P et al (1996) Human methionine synthase: cDNA cloning and identification of mutations in patients of the cblG complementation group of folate/cobalamin disorders. Hum Mol Genet 5:1867-1874

Leclerc D, Wilson A, Dumas R et al (1998) Cloning and mapping of a cDNA for methionine synthase reductase, a flavoprotein defective in patients with homocystinuria. Proc Natl Acad Sci U S A 95: 3059-3064

Lerner-Ellis JP, Tirone JC, Pawelek PD et al (2006) Identification of the gene responsible for methylmalonic aciduria and homocystinuria, cblC type. Nat Genet 38:93-100

Li YN, Gulati S, Baker PJ, Brody LC, Banerjee R, Kruger WD (1996) Cloning, mapping and RNA analysis of the human methionine synthase gene. Hum Mol Genet 5:1851-1858

Mah W, Deme JC, Watkins D et al (2013) Subcellular location of MMACHC and MMADHC, two human proteins central to intracellular vitamin B(12) metabolism. Mol Genet Metab 108:112-118 
Mahoney MJ, Rosenberg LE (1971) Synthesis of cobalamin coenzymes by human cells in tissue culture. J Lab Clin Med 78:302-308

Nicholas KB, Nicholas HBJ, Deerfield DW II (1997) GeneDoc: analysis and visualization of genetic variation. EMBNEW.NEWS: 14

Parini R, Furlan F, Brambilla A et al (2013) Severe neonatal metabolic decompensation in methylmalonic acidemia caused by CblD defect. JIMD Rep 11:133-137

Plesa M, Kim J, Paquette SG et al (2011) Interaction between MMACHC and MMADHC, two human proteins participating in intracellular vitamin B(1)(2) metabolism. Mol Genet Metab 102:139-148
Rutsch F, Gailus S, Miousse IR et al (2009) Identification of a putative lysosomal cobalamin exporter altered in the cblF defect of vitamin B12 metabolism. Nat Genet 41:234-239

Stucki M, Coelho D, Suormala T, Burda P, Fowler B, Baumgartner MR (2012) Molecular mechanisms leading to three different phenotypes in the cblD defect of intracellular cobalamin metabolism. Hum Mol Genet 21:1410-1418

Suormala T, Baumgartner MR, Coelho D et al (2004) The cblD defect causes either isolated or combined deficiency of methylcobalamin and adenosylcobalamin synthesis. J Biol Chem 279:42742-42749 